\title{
Petiscos florais: pétalas de Acca sellowiana (Myrtaceae) como fonte alimentar para aves em área urbana no Sul do Brasil
}

\author{
Ivan Sazima ${ }^{1,3}$ \& Marlies Sazima ${ }^{2}$ \\ Biota Neotropica $v 7(n 2)$ \\ http://www.biotaneotropica.org.br/v7n2/pt/abstract?short-communication+bn01907022007 \\ Recebido em 16/01/07 \\ Versão reformulada recebida em 08/04/07 \\ Publicado em 01/05/07 \\ ${ }^{1}$ Departamento de Zoologia e Museu de História Natural, \\ Universidade Estadual de Campinas - UNICAMP, CP 6109, CEP 13083-970, Campinas, São Paulo, Brasil \\ ${ }^{2}$ Departamento de Botânica, Universidade Estadual de Campinas - UNICAMP, \\ CP 6109, CEP 13083-970, Campinas, São Paulo, Brasil \\ ${ }^{3}$ Autor para correspondência: Ivan Sazima, e-mail: isazima@gmail.com,www.unicamp.br
}

\begin{abstract}
Sazima, I. and Sazima, M. Floral titbits: petals of Acca sellowiana (Myrtaceae) as a food source for birds in an urban area in Southern Brazil. Biota Neotrop. May/Aug 2007 vol. 7, no. 2 http://www.biotaneotropica. org.br/v7n2/pt/abstract?short-communication+bn01907022007 ISSN 1676-0603.

The feijoa (Acca sellowiana, Myrtaceae) is one of the few plant species whose petals are habitually eaten by birds in the Neotropics. We record here the use of petals of this myrtaceous plant by nine species of passerine birds of an isolated plant in the urban area of Gramado, Rio Grande do Sul, southern Brazil. Highest visiting frequency was recorded for tanagers (Thraupidae), a predominantly frugivorous bird group. The birds took the petals within their mandibles and tore or cut pieces off. Thraupis sayaca and Tangara preciosa were recorded collecting several petal pieces with their mandibles, the first species being observed twice feeding petals to nestlings. Due to its medium size and the showy flowers, as well as to its attractiveness to several bird species even in an urbane area, we reiterate here the recommendation to use A. sellowiana in parks and gardens in southern and southeastern Brazil.
\end{abstract}

Keywords: floral resources, urbane areas, Passeriformes, urbane arborisation.

Resumo

Sazima, I. and Sazima, M. Petiscos florais: pétalas de Acca sellowiana (Myrtaceae) como fonte alimentar para aves numa área urbana no Sul do Brasil. Biota Neotrop. May/Aug 2007 vol. 7, no. 2 http://www. biotaneotropica.org.br/v7n2/pt/abstract?short-communication+bn01907022007 ISSN 1676-0603.

A goiaba-serrana (Acca sellowiana, Myrtaceae) é uma das poucas espécies vegetais cujas pétalas são habitualmente usadas como alimento por aves nos Neotrópicos. Registramos aqui o consumo de pétalas desta mirtácea por nove espécies de aves Passeriformes, numa arvoreta isolada em área urbana de Gramado, Rio Grande do Sul. A maior freqüência de visitas foi registrada para espécies de Thraupidae, aves predominantemente frugívoras. As aves tomavam as pétalas entre as mandíbulas e arrancavam ou cortavam porções, deixando sinais característicos nas flores. Thraupis sayaca e Tangara preciosa foram registrados recolhendo porções de pétalas e levando-as entre as mandíbulas, a primeira espécie tendo sido duas vezes observada fornecendo pétalas a ninhegos. Devido ao seu porte médio e à beleza das suas flores, bem como à atração de diversas espécies de pássaros mesmo em área urbanizada, reiteramos aqui o uso de A. sellowiana em arborização urbana no Sul e Sudeste do Brasil.

Palavras-chave: recursos florais, áreas urbanizadas, Passeriformes, arborização urbana. 


\section{Introdução}

Pétalas de flores de poucas espécies de plantas neotropicais são fonte alimentar para aves outras que psitacídeos (periquitos e papagaios), os quais se alimentam destas partes florais habitualmente (e.g. Galetti 1993, Sick 1997). Um exemplo notável é o consumo de partes da corola de Calceolaria uniflora (Scrophulariaceae) por Thinocorus rumicivorus (Thinocoridae), na Argentina (Sérsic \& Cocucci 1996). Um outro exemplo é o consumo das pétalas de duas espécies de Myrtaceae, Myrrhinium atropurpureum e Acca sellowiana (Roitman et al. 1997), ambas restritas à América do Sul (Landrum 1986, Marchiori \& Sobral 1997, Piedrabuena 2004) e visitadas principalmente por aves Passeriformes (Roitman et al. 1997, Ducroquet $\&$ Hickel 1997). Nenhuma destas duas espécies produz néctar e seu pólen é procurado por insetos (revisão em Gressler et al. 2006).

O consumo de pétalas de A. sellowiana por aves está registrado na literatura científica desde o final do século 19 (Knuth 1898). Os estudos sobre a biologia desta espécie, de valor ornamental e comercial (Stewart \& Craig 1989, Patterson 1990, Lorenzi 1992, Free 1993), estão restritos a poucos relatos sobre plantas cultivadas na Nova Zelândia e no Brasil (revisão em Gressler et al. 2006). A ampla difusão da informação de que as pétalas de A. sellowiana sejam consumidas por aves (e.g., Stewart \& Craig 1989, Roitman et al. 1997, Gressler et al. 2006) fornece a falsa idéia de que a relação desta espécie de mirtácea com as aves visitantes seja bem conhecida, o que está longe de ser verdadeiro, pois os poucos dados disponíveis (e.g. Knuth 1898, Stewart \& Craig 1989, Ducroquet \& Hickel 1997) têm sido meramente repetidos de um estudo para outro.

Registramos aqui o consumo de pétalas desta mirtácea por aves Passeriformes, numa arvoreta isolada em área urbana de Gramado, Rio Grande do Sul. O presente estudo constitui um instantâneo ("snapshot") do uso das flores de A. sellowiana por aves que vivem em áreas urbanas. As principais questões enfocadas neste estudo de breve duração foram: (1) Quais espécies de aves podem ser registradas num indivíduo isolado de A. sellowiana em área urbana? (2) Qual sua frequiência relativa? (3) Qual é o seu comportamento alimentar nas flores? Uma arvoreta isolada, caso visitada, indicaria a importância desta mirtácea como fonte alimentar para aves que vivem em áreas urbanizadas e forneceria incentivo adicional ao uso de A. sellowiana na arborização de áreas urbanas no Sul e no Sudeste do Brasil.

\section{Material e Métodos}

As observações foram feitas no bairro Carniel, a quatro km do centro de Gramado (ca. $29^{\circ} 23^{\prime}$ S e $51^{\circ} 06^{\prime}$ W; 900 m a.n.m.), Rio Grande do Sul. O indivíduo observado de A. sellowiana era uma arvoreta ca. $3 \mathrm{~m}$ de altura, num jardim defronte a rua asfaltada (Figura 1a) com trânsito moderado de pedestres e veículos (máximo de 20 pessoas e 8 veículos/h). Nos arredores do bairro havia alguns remanescentes de mata nativa, com evidentes sinais de perturbação antropogênica. Entre as árvores de maior porte havia principalmente Podocarpus sellowii (Podocarpaceae) e alguns indivíduos de Araucaria angustifolia (Araucariaceae).

A arvoreta de goiaba-serrana foi observada em diferentes horários, ao longo de quatro dias (7 a 10 de novembro de 2006), cobrindo diversos períodos do dia entre 0640 e 1830 h, num total de 755 min de observação focal (Lehner 1979). As flores eram examinadas ao início e ao final de cada sessão de observação, para detectar os períodos de antese (cf. Faegri \& van der Pijl 1980). As pétalas de flores, em diversas fases de antese, foram testadas organolepticamente (mastigadas e saboreadas). Embora rudimentar e sujeito a variação de percepção individual, o teste permite detectar mudanças de sabor durante as fases de antese. Durante as sessões de observação (20-155 min/sessão), cada ave visitante foi observada com auxílio de
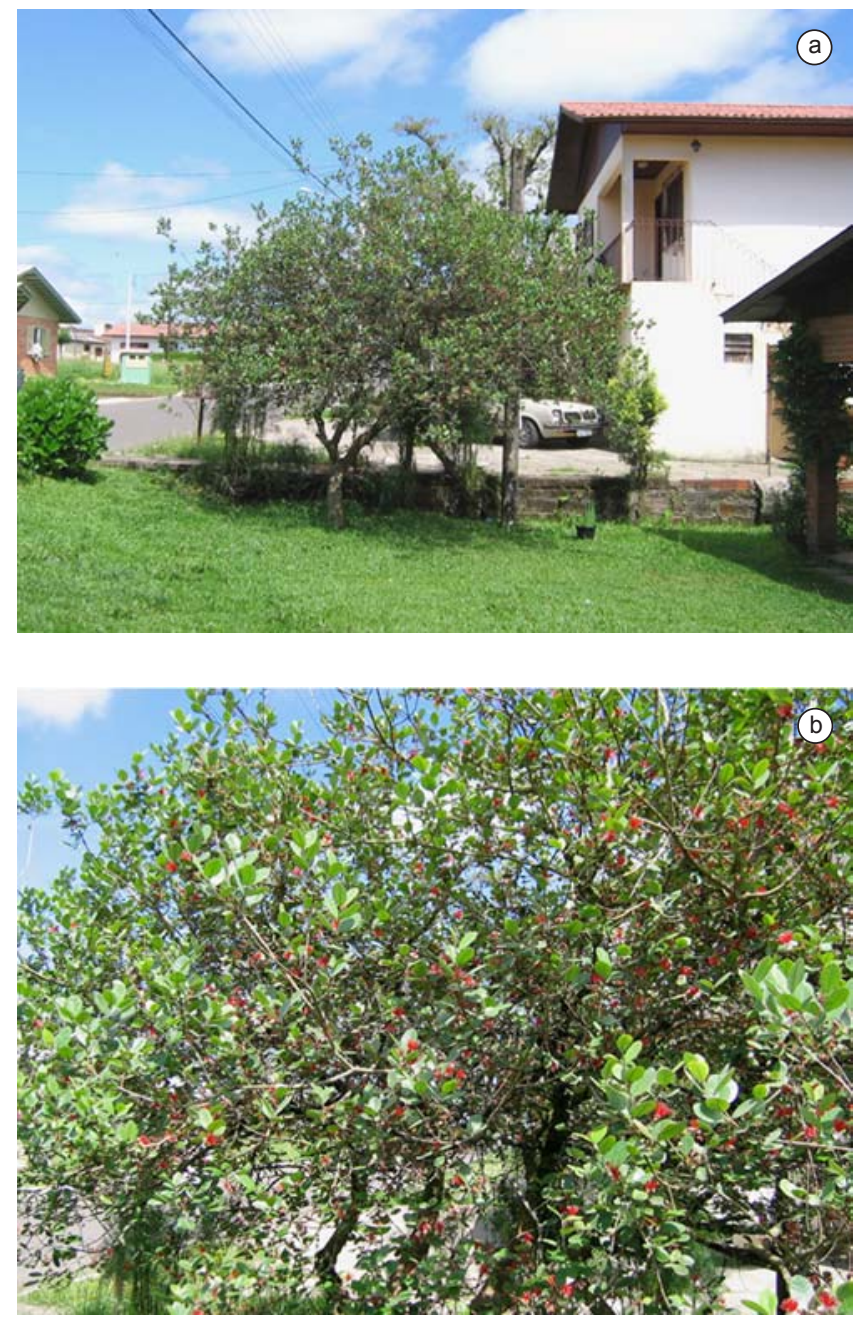

Figura 1. a) Localização; e b) ramos floridos da arvoreta de Acca sellowiana observada em área urbana de Gramado, Rio Grande do Sul.

Figure 1. a) Location and; b) blooming branches of the small tree of Acca sellowiana plant observed in urban area at Gramado, Rio Grande do Sul state, southern Brazil.

binóculo, sendo a identidade ou as características diagnósticas anotadas em caderno de campo e posteriormente conferidas com auxílio de guias de campo e textos ornitológicos (e.g. Dunning 1982, Sick 1997, Narosky \& Yzurieta 2006). No caso de espécies com dimorfismo sexual evidente, foi também anotado o sexo da ave visitante. O comportamento de forrageio das aves nas flores foi registrado e os vestígios decorrentes da alimentação foram fotografados quando inequivocamente associados a determinada ave. Visitas de insetos às flores não foram registradas. A classificação e os nomes científicos das aves seguem o CBRO (2006).

\section{Resultados}

No período do estudo, a arvoreta de A. sellowiana estava em plena florada (Figura 1b), com cerca de 150 flores abertas diariamente. A abertura de novas flores ocorria nos mais diversos períodos do dia, desde a manhã até o entardecer, havendo sempre flores em processo de abertura ao lado de flores totalmente abertas e com pólen disponível e outras, com pétalas bicadas e/ou murchas e sem pólen (Figura 2a, b). Ao serem mastigadas, as pétalas das flores em estágios iniciais de abertura eram levemente ácidas, sabor que se tornava mais adocicado 
à medida do avanço dos estágios de antese. Pétalas de flores com pólen disponível nas anteras eram as de sabor mais doce.

Cento e cinquienta indivíduos de aves Passeriformes (11,92 indivíduos/h) foram registrados consumindo pétalas de A. sellowiana na arvoreta durante o breve período de estudo, somando nove espécies distribuídas em quatro famílias e sete gêneros (Tabela 1). Espécies de Thraupidae foram os visitantes registrados com maior frequiência, com predomínio de três espécies, Thraupis sayaca, Tangara preciosa e Stephanophorus diadematus, cada uma com freqüência relativa acima de $10 \%$ (Tabela 1). Com exceção de Thraupis bonariensis, cuja freqüência relativa foi $7,33 \%$, as demais cinco espécies foram registradas muito ocasionalmente $(4,64 \%$ do total).
As aves tomavam as pétalas entre as mandíbulas e faziam movimentos de puxar ou "cortar" (mandibular), retirando porções das pétalas e deixando sinais característicos nas flores (Figuras $2 \mathrm{~b}$ e 3c). A maioria das aves, em particular Tangara preciosa e Thraupis sayaca (registradas com maior precisão devido ao maior número de observações) retirava porções da ponta ou da borda das pétalas (Figura 2b). Por outro lado, Stephanophorus diadematus (Figura 3b) tomava a pétala transversalmente entre as mandíbulas e retirava a maior parte (Figura 3c). Em geral a porção retirada era ingerida em seguida, passando a ave a retirar outra porção, da mesma ou de outra flor. Ao retirar porções das pétalas, as aves contatavam os elementos reprodutivos das flores com partes da cabeça e do pescoço, principalmente. Em nenhuma ocasião foram registrados danos às porções
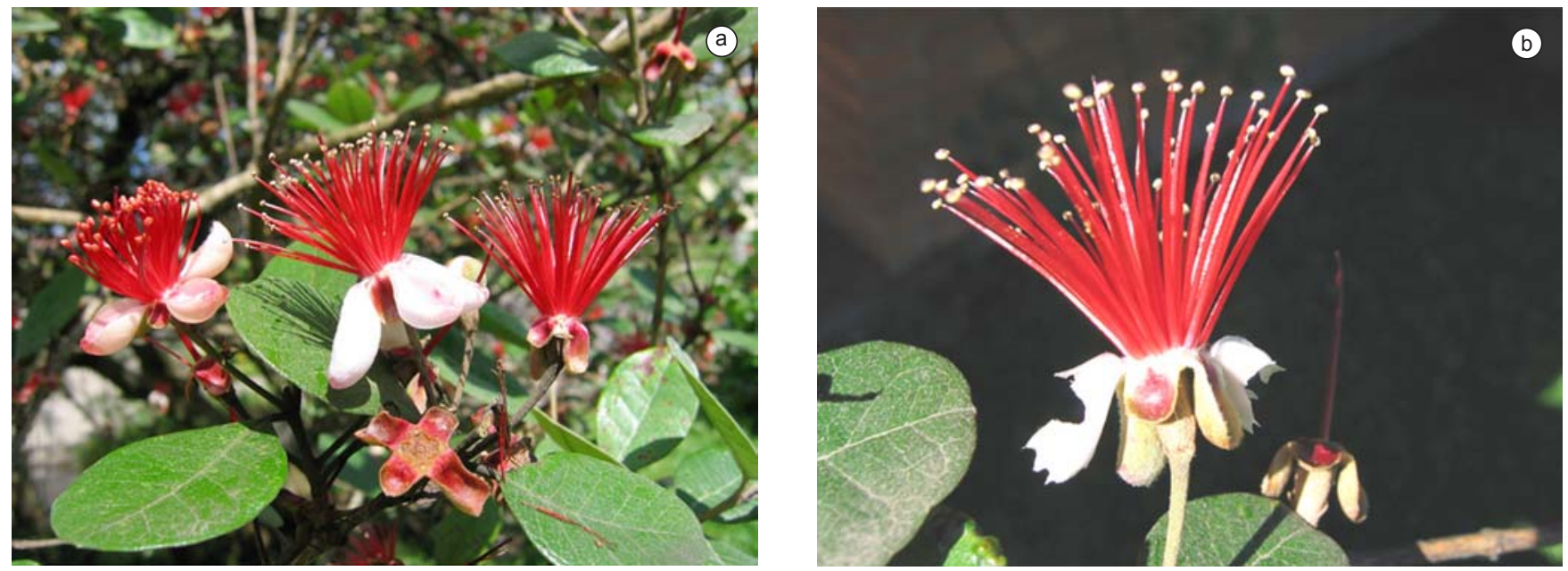

Figura 2. a) Flores de Acca sellowiana em três fases de antese no mesmo ramo; e b) flor com porções retiradas por aves, com sinais correspondentes às mandíbulas nas bordas das pétalas.

Figure 2. a) Acca sellowiana flowers in three phases of anthesis on the same branch; and b) a flower with portions removed by birds, with bill marks on the petals' edges.

Tabela 1. Espécies de aves Passeriformes registradas consumindo pétalas de Acca sellowiana numa arvoreta isolada em área urbana de Gramado, Rio Grande do Sul, ao longo de quatro dias em novembro de 2006. A sequiência de famílias segue o CBRO (2006); gêneros e espécies em ordem alfabética. Número (N) e proporção relativa (\%) de visitas registradas; número de visitas de machos e fêmeas ( $\delta /$ ) de três espécies com dimorfismo sexual evidente; itens alimentares predominantes na dieta (cf. Argel-de-Oliveira \& Figueiredo 1996, Sick 1997, Develey \& Endrigo 2004).

Table 1. Species of passerine birds recorded feeding on Acca sellowiana petals on an isolated tree in urban area at Gramado, Rio Grande do Sul state, southern Brazil, over four days in November 2006. Sequence of families follows the CBRO (2006); genera and species in alphabetical order. Number (N) and relative proportion (\%) of recorded visits; number of visits by males and females ( $\delta / q)$ of three species with evident sexual dimorphism; predominant food items in the diet (cf. Argel-de-Oliveira \& Figueiredo 1996, Sick 1997, Develey \& Endrigo 2004).

\begin{tabular}{|c|c|c|c|}
\hline Espécies & $\mathbf{N}(\%)$ & $3 / 9$ & Dieta \\
\hline \multicolumn{4}{|l|}{ Tyrannidae } \\
\hline Elaenia parvirostris & $2(1,33)$ & - & Artrópodes/frutos \\
\hline \multicolumn{4}{|l|}{ Turdidae } \\
\hline Turdus amaurochalinus & $1(0,66)$ & - & Invertebrados/frutos \\
\hline Turdus rufiventris & $1(0,66)$ & - & Invertebrados/frutos \\
\hline \multicolumn{4}{|l|}{ Thraupidae } \\
\hline Dacnis cayana & $2(1,33)$ & $0 / 2$ & Frutos/artrópodes/néctar \\
\hline Stephanophorus diadematus & $20(13,33)$ & - & Frutos/artrópodes/flores \\
\hline Tangara preciosa & $30(20)$ & $19 / 11$ & Frutos/artrópodes \\
\hline Thraupis bonariensis & $11(7,33)$ & $6 / 5$ & Frutos/artrópodes \\
\hline Thraupis sayaca & $82(54,66)$ & - & Frutos/flores/artrópodes \\
\hline \multicolumn{4}{|l|}{ Emberizidae } \\
\hline Zonotrichia capensis & $1(0,66)$ & - & Sementes/artrópodes \\
\hline
\end{tabular}





Figura 3. a) Flor de Acca sellowiana com marca de mandíbula (da esquerda para a direita) na pétala em primeiro plano, sem retirada de qualquer porção; b) Stephanophorus diadematus, traupídeo característico de florestas montanas, e c) sinais deixados na flor pelo seu bico forte.

Figure 3. a) Flower of Acca sellowiana with a bill mark (from the left to the right) on the petal in the foreground, with no portion removed; b) Stephanophorus diadematus, a distinctive, montane forests-dwelling thraupid; and c) marks left on a flower by its strong bill. reprodutivas das flores, embora alguns dos numerosos estames possam ser deslocados das flores durante a remoção das pétalas.

Diversos indivíduos de $T$. sayaca e $S$. diadematus foram observados fechando as mandíbulas sobre algumas pétalas, porém sem retirar qualquer porção. Essas pétalas apresentavam marcas do contorno da mandíbula, resultantes do contato do bico da ave (Figura 3a).

Thraupis sayaca e Tangara preciosa foram observadas colhendo e reunindo porções de pétalas entre as mandíbulas, visitando até 4-5 flores em seguida. Após a colheita, retiravam-se carregando as pétalas no bico. Defronte à arvoreta da goiaba-serrana, do lado oposto da rua, havia um indivíduo de cipreste (Cupressaceae), no qual foi percebido um ninho de T. sayaca com dois ou três filhotes. Em duas ocasiões, um dos adultos foi observado alimentando os filhotes com pétalas da goiaba-serrana, que havia acabado de colher.

\section{Discussão}

Acca sellowiana floresce entre os meses de outubro e janeiro (Lorenzi 1992); portanto, a florada da arvoreta por nós estudada em área urbana coincide bem com registros da literatura. A presença simultânea de flores abertas em diferentes fases de antese, aqui observada para A. sellowiana, também é conhecida para Myrrhinium atropurpureum, a única outra mirtácea cujas pétalas são habitualmente consumidas por aves (Roitman et al. 1997). A mudança de sabor detectada nas pétalas de A. sellowiana em diferentes fases de antese é mencionada por Knuth (1898). Nas flores de Myrrhinium atropurpureum, há variação na concentração de açúcares e quantidade de água nas pétalas durante a antese (Roitman et al. 1997). A mandibulação das pétalas pelas aves poderia ser interpretada como um tipo de "prova organoléptica" que as aves fariam para escolher as pétalas mais doces e, portanto, mais energéticas.

O elenco de aves visitantes das flores de A. sellowiana, registrado no presente estudo, é semelhante ao relatado para plantas cultivadas na região de Videira, Santa Catarina, com presença de Thraupidae, Turdidae e Tyrannidae (Ducroquet \& Hickel 1997). Embora estes autores sejam genéricos em boa parte das suas identificações, algumas espécies como Thraupis sayaca, T. bonariensis, Stephanophorus diadematus e Turdus rufiventris, também foram registradas nas plantas cultivadas (Ducroquet \& Hickel 1997). Pelo menos oito espécies foram registradas por Ducroquet \& Hickel (1997), número comparável ao observado na área urbana por nós estudada, ainda que numa arvoreta isolada. Em flores de $M$. atropurpureum na Província de Entre Rios, Argentina (Roitman et al. 1997), foram registradas 14 espécies de aves, cuja dieta foi caracterizada em quatro categorias: frutos/invertebrados (cinco espécies), invertebrados/frutos (5), sementes (3), invertebrados (1). Composição semelhante de dieta foi aqui registrada para as aves visitantes da arvoreta de $A$. sellowiana em área urbana (Tabela 1), com predomínio de espécies que se nutrem de frutos e invertebrados (cinco espécies). Dada a variedade da dieta, as aves visitantes às flores de $A$. sellowiana são comparáveis àquelas que visitam plantas com frutos de pequeno porte como Ficus (Moraceae) ou Miconia e Leandra (Melastomataceae), as quais são visitadas principalmente por aves onívoras e frugívoras não-especializadas (Argel-de-Oliveira \& Figueiredo 1996, Griddi-Papp et al. 2004).

A predominância de espécies de Thraupidae (gêneros Tangara, Thraupis, Stephanophorus), entre as aves registradas nas flores de A. sellowiana, está relacionada à dieta destas aves, que se alimentam de frutos, folhas e flores, incluindo pétalas e néctar (Sazima et al. 1993, Sick 1997, Develey \& Endrigo 2004). A maior freqüência de Thraupis sayaca reflete a adaptação desta espécie aos ambientes urbanizados (e.g. Sick 1997, Develey \& Endrigo 2004). Stephanophorus diadematus e Tangara preciosa estão entre as espécies típicas de regiões serranas (Sick 1997, Develey \& Endrigo 2004, Narosky \& 
Yzurieta 2006) e a sua presença na área urbana de Gramado provavelmente está relacionada aos fragmentos florestais, ainda que antropicamente perturbados. Thraupis bonariensis, uma espécie meridional, habita capões e borda de matas (Sick 1997, Narosky \& Yzurieta 2006), o que possivelmente facilita sua presença em áreas urbanas. Elaenia parvirostris também é uma espécie do Brasil meridional, migratória, que nidifica no Rio Grande do Sul à época das nossas observações (Sick 1997).

Embora na literatura conste a informação de que as pétalas de A. sellowiana sejam consumidas por aves (e.g. Lughadha \& Proença 1996, Roitman et al. 1997, Ducroquet \& Hickel 1997), não há menção ao modo pelo qual esse recurso seja removido das flores. Observamos que as pétalas não são retiradas inteiras, como o termo "remover" faria supor, porém porções delas são recortadas e/ou arrancadas de acordo com a espécie de ave, uma informação nova na relação entre as aves e esta espécie de mirtácea. As aves visitantes apresentam potencial para agir como polinizadores de A. sellowiana (veja também Ducroquet \& Hickel 1997); porém, a sua função na polinização desta espécie em seu habitat natural permanece sem confirmação adequada.

O período de floração de A. sellowiana na área do estudo coincide com a nidificação e o cuidado à prole de algumas das aves aqui registradas (Sick 1997, obs. pess.). Acreditamos que as pétalas representem um recurso energético adicional, talvez comparável à polpa de alguns frutos, à dieta dos ninhegos. Além disso, familiariza os juvenis com esse tipo de fonte alimentar. O fornecimento das pétalas de A. sellowiana a ninhegos de Thraupis sayaca (e, presumivelmente, também de Tangara preciosa) é um dos registros inéditos do presente estudo e indica o pouco conhecimento disponível sobre a relação desta mirtácea com as aves visitantes.

Devido ao seu porte médio e à beleza das suas flores, bem como à atração de diversas espécies de pássaros (que também se alimentam dos seus frutos maduros, Lorenzi 1992), mesmo em áreas urbanizadas, reiteramos aqui a recomendação do uso de A. sellowiana em arborização urbana no Sul e Sudeste do Brasil. Acreditamos que esta mirtácea ficaria aclimatizada mesmo em regiões como o interior de São Paulo, pois temos conhecimento de indivíduos desta espécie cultivados no Instituto Agronômico de Campinas (obs. pess.). Seu cultivo, desenvolvido com sucesso na Nova Zelândia (e.g. Stewart \& Craig 1989, Patterson 1990), tem sido negligenciado no Brasil (Romero 2007). Mesmo seu crescimento lento (Lorenzi 1992) não deveria desestimular o uso de A. sellowiana em arborização urbana ou, ao menos, em jardins e quintais.

\section{Agradecimentos}

Aos moradores do bairro Carniel, que gentilmente toleraram a nossa presença defronte às suas residências nos mais diversos horários; a Robson Silva e Silva pela imagem de Stephanophorus diadematus; ao CNPq pelo indispensável auxílio financeiro.

\section{Referências Bibliográficas}

ARGEL-DE-OLIVEIRA, M.M. \& FIGUEIREDO, R.A. 1996. Aves que visitam uma figueira isolada em ambiente aberto, Espírito Santo, Brasil. Iheringia, Ser. Zool. 80:127-134.

COMITÊ BRASILEIRO DE REGISTROS ORNITOLÓGICOS (CBRO) Lista das aves do Brasil. Versão 10/2/2006 <http://www.cbro.org.br> (último acesso em janeiro 2007).
DEVELEY, P.F. \& ENDRIGO, E. 2004. Aves da Grande São Paulo, guia de campo. Aves e Fotos Editora, São Paulo.

DUCROQUET, J.P.H.J. \& HICKEL, E.R. 1997. Birds as pollinators of Feijoa (Acca sellowiana Berg.). Acta Hort. 452:37-40.

DUNNING, J.S. 1982. South American land birds, a photographic aid to identification. Harrowood Books, Pennsylvania.

FAEGRI, K. \& VAN DER PIJL, L. 1980. The principles of pollination ecology. $2^{\text {nd }}$ ed. Pergamon Press, New York.

FREE, J.B. 1993. Insect pollination of crops. Academic Press, London.

GALETTI, M. 1993. Diet of the scaly-headed parrot (Pionus maximiliani) in a semi-deciduous forest in southeastern Brazil. Biotropica. 25(4):419-425.

GRESSLER, E., PIZO, M.A. \& MORELLATO, P.C. 2006. Polinização e dispersão de sementes em Myrtaceae do Brasil. Rev. Bras. Bot. 29(4):509-530.

GRIDDI-PAPP, C.O., GRIDDI-PAPP, M. \& RODRIGUES, W.R. 2004. Differential fruit consumption of two Melastomataceae by birds in Serra da Mantiqueira, southeastern Brazil. Ararajuba 12(1):5-10.

KNUTH, P. 1898. Handbuch der Blütenbiologie. Verlag Wilhelm Engelmann, Leipzig.

LANDRUM, L.R. 1986. Campomanesia, Pimenta, Blepharocalyx, Legrandia, Acca, Myrrhinium, and Luma (Myrtaceae). Fl. Neotrop.Monogr. 45. New York Botanical Garden, New York.

LEHNER, P.N. 1979. Handbook of ethological methods. Garland STPM, New York.

LORENZI, H. 1992. Árvores brasileiras: Manual de identificação e cultivo de plantas arbóreas nativas do Brasil. Editora Plantarum Ltda., Nova Odessa.

LUGHADHA, E.N. \& PROENÇA, C. 1996. A survey of the reproductive biology of the Myrtoideae (Myrtaceae). Ann. Missouri Bot. Gard. 83:480-503.

MARCHIORI, J.N.C. \& SOBRAL, M. 1997. Dendrologia das angiospermas: Myrtales. Editora Universidade Federal de Santa Maria, Santa Maria.

NAROSKY, T. \& YZURIETA, D. 2006. Birds of Argentina \& Uruguay, a field guide. $15^{\text {th }}$ ed. Vazquez Mazini Editores, Buenos Aires.

PATTERSON, K.J. 1990. Effects of pollination on fruit set, size, and quality in feijoa (Acca sellowiana) (Berg.) Burret). N. Z. J. Crop Hort. Sci. 18:127-131.

PIEDRABUENA, F.P. 2004. Flora Nativa. Arboles y arbustos del Uruguay y regiones vecinas. Guía de campo y usos medicinales. Tomo I. Ediciones Guyunusa, Maldonado.

ROITMAN, G.G, MONTALDO, N.H. \& MEDAN, D. 1997. Pollination biology of Myrrhinium atropurpureum (Myrtaceae): sweet fleshy petals attract frugivorous birds. Biotropica 29(2):162-168.

ROMERO, T. 2007. Plantas do futuro. Agência de notícias da Fundação de Amparo à Pesquisa do Estado de São Paulo. http://www.agencia.fapesp. br/boletim_print.php?data[id_material_boletim]=6582. (Acesso em 10/01/2007).

SAZIMA, I., BUZATO, S. \& SAZIMA, M. 1993. The bizarre inflorescence of Norantea brasiliensis (Marcgraviaceae): visits of hovering and perching birds. Bot. Acta 106(6):507-513.

SÉRSIC, A.N. \& COCUCCI, A.A. 1996. A remarkable case of ornithophily in Calceolaria: food bodies as rewards for a non-nectarivorous bird. Bot. Acta 109(2):172-176.

SICK, H. 1997. Ornitologia brasileira. Editora Nova Fronteira, Rio de Janeiro.

STEWART, A.M. \& CRAIG, J.L. 1989. Factors affecting pollinator effectiveness in Feijoa sellowiana. N. Z. J. Crop Hort. Sci. 17:145-154. 